\title{
Renewable energy EV charging station
}

\author{
Petreus Dorin, Patarau Toma, Etz Radu \\ Department of Applied Electronics \\ Faculty of Electronics, Telecommunications and Information \\ Technology, Technical University of Cluj-Napoca, \\ Cluj-Napoca, Romania \\ dorin.petreus@ael.utcluj.ro
}

\author{
Cirstea Marcian \\ School of Computing and Information Science, \\ Faculty of Science and Engineering \\ Anglia Ruskin University \\ Cambridge, United Kingdom \\ marcian.cirstea@anglia.ac.uk
}

\begin{abstract}
Due to the increased interest in electric vehicle (EV) technologies and the cost reduction of photovoltaic systems, industrial development of battery charging stations for electric vehicles based on solar energy has started. Although it is convenient to charge EVs at night, when there is less electricity consumption, there will always be consumers who prefer or need to charge their $\mathrm{EV}$ during daytime and even during peak hours. In this paper, an EV charging station integrating renewable energy in the form of solar energy is proposed and analyzed. Using a local battery pack, the charging station allows semi-fast and fast charging and can be installed in individual homes and apartment buildings, where the maximum power delivered by the grid is limited to $3.6 \mathrm{~kW}$. The charging station uses converters widely available on the market. A simulation model for this charging station is developed and various distinct operation modes are presented, validating the correct operation of the entire system.
\end{abstract}

\section{Keywords - charging station, electric vehicle, solar energy}

\section{INTRODUCTION}

The modern Electric Vehicle (EV) technologies are relatively new and EVs are starting to gain popularity due to their numerous advantages: zero emissions, reduced fossil fuels use, efficiency, low noise, and so on. Environmental pollution, depletion of fossil fuels, growing consumer expectations and increased fuel costs lead manufacturers (ABB, SMA, Siemens and others) to start focusing on EV, battery chargers and charging stations. Nowadays, research on electric vehicles is focusing on increasing the autonomy and efficiency of vehicles, reducing prices, and developing methods for an efficient charging system [1]-[6] .

There are three main authorities that issue standards for EV battery charging: IEC, SAE, and CHAdeMO. In addition to these, Tesla has proposed its own standard for charging their EVs [7]. According to SAE J1772 standard, the charging systems for EVs are categorized based on different power levels. These levels are summarized in Table 1.

TABLE I. CHARGER TYPES

\begin{tabular}{lllll}
\hline Types & Level & Charger type & $\begin{array}{l}\text { Power } \\
\text { level }\end{array}$ & $\begin{array}{l}\text { Charging } \\
\text { time }\end{array}$ \\
\hline \multirow{3}{*}{ AC } & Level 1 & On-board & $1.4 \mathrm{~kW}$ & $14-17 \mathrm{~h}$ \\
\cline { 2 - 5 } & Level 2 & On-board & $4 \mathrm{~kW}$ & $4-6 \mathrm{~h}$ \\
\cline { 2 - 5 } & Level 3 & To be decided & $>20 \mathrm{~kW}$ & $0.4-1 \mathrm{~h}$ \\
\hline \multirow{3}{*}{ DC } & Level 1 & Off-board & $36 \mathrm{~kW}$ & $0.4-1 \mathrm{~h}$ \\
\cline { 2 - 5 } & Level 2 & Off-board & $90 \mathrm{~kW}$ & $10-20 \mathrm{~min}$ \\
\cline { 2 - 5 } & Level 3 & Off-board & $240 \mathrm{~kW}$ & $<10 \mathrm{~min}$ \\
\hline
\end{tabular}

AC Level 1 is the most used household slow charging method. This mode is suitable for recharging electrical battery packs during the night, requiring low power consumption from the grid. AC level 2 is a semi-fast charging method which requires $4 \mathrm{~kW}$ of power for charging the battery. $\mathrm{AC}$ Level 3 is a three-phase charging method. EV chargers are classified as on-board and off-board chargers [8]. All the DC charging methods are fast charging and use off-board charging stations.

This interaction between the EV, the households and the grid will demand efforts oriented towards developing smart energy management strategies facilitating consumers comfort, reduced costs, improved energy quality and energy efficiency [9]-[11]. There are several problems related to the connection of the electric vehicle to the grid: voltage instability, voltage sag, voltage unbalance, overloading, harmonics, frequency variation, energy losses, voltage unbalance and others [9]. All these problems related to EV penetration into the grid lead to reductions in power quality. The load of the charging station is significant and can very easily overload the grid. Due to increased EV charging, the local distribution network (feeders, cables, distribution transformers, substation equipment) may experience high stress levels and increased heating. Grid constraints can also be exceeded. Because of the huge electricity demand, the grid voltage may drop to low values causing voltage instabilities and voltage dips. Appliances connected to the grid may be damaged due to fluctuations and grid instability [12]. The converters commonly used in EV charging stations can cause excessive harmonics injection into the grid. Among other issues, harmonics lead to poor power quality due to distortion of waveforms, stress on power system equipment and currents on the neutral cables. Voltage imbalance is a serious problem affecting three-phase systems and occurs when there is an unbalanced load connected to the lines of the three-phase system [13]. The lack of experimental data for EV charging applications justifies the need of laboratory experiments using small-scale prototypes.

\section{Motivation}

The electricity network can be transformed from the current form of unidirectional "rigid" network into a dynamic network that allows adaptation to energy requirements by operating bidirectionally and providing the necessary energy on demand. Given that most home users have access to a single-phase network, it is necessary to introduce additional power generators to work in tandem with the grid. Further developments require the integration of information technologies in energy production and distribution. From this point of view, the electric vehicle should no longer be seen by the network only as a passive consumer but as an active 
element that allows the expansion of energy storage and transport capacity.

The most debated issue so far regarding electric vehicles has been that of the reduced autonomy it offers compared to classic cars. The more the number of charging stations increases and the more efficiently they are used, the shorter their integration time into society could be. One way to achieve high performance on short-term charging and reduce charging costs is to use the single-phase network in a hybrid configuration with small energy production systems based on renewable energy (solar, wind, geothermal, biomass, hydrogen) in combination with high capacity storage systems [14]-[19].

Although it is convenient to charge the electrical vehicles at night, when there is less electricity consumption, there will always be consumers who prefer or need to charge their EV during daytime and even during peak hours. Transmission and distribution networks can be upgraded to meet the peak demand but usually this results in capacity surplus during normal hours and at night. Also, transformers with higher capacity are expensive to replace. An alternative is to develop EV charging stations coupled with smart microgrids that use renewable energies. The maximum amount of power that can be extracted from the main grid is generally constant and much lower than the power needed for fast charging of the electric vehicle battery pack. Furthermore, semi-fast and fast charging stations can be installed in individual homes and apartment buildings. In this case, the maximum allowed power can be extracted from the grid and the remaining power needed will be supplied by the renewable energy sources. It is estimated that vehicles are parked for at least $5 \mathrm{~h}$ in the workplace environment, hence these places are also favorable for renewable energy charging station development.

Due to the recent photovoltaic (PV) system price decreases, government initiatives and subsidies, photovoltaic energy would be a good choice to supplement power to the charging station. Due to the intermittent nature of the solar energy, the PV system can be successfully used in combination with external storage. Because the storage is usually expensive, proper sizing of the storage system and energy management algorithms that also account for weather forecast data are essential. Not many studies were reported related to the case of reducing the loading on the distribution grid due to EV chargers using photovoltaic systems. Photovoltaic energy is a feasible solution, especially for daytime charging [7]. Furthermore, if the charging takes place during peak hours, when the cost of electricity is the highest, then substantial economic benefits can be rendered. The main advantages expected from such a PV-battery-charging station are: reduced energy demand from the grid (as power is locally generated), the energy storage reduces the negative effects of PV integration in the grid, possibilities of implementation of vehicle to home (V2H) technologies [4], [20]. Romania has met its EU targets on renewable energy generation in 2015, far ahead the 2020 target deadline, making EV charging [21] a feasible endeavor.

Considering all the above, the novelty of the paper is the analysis of a proposed charging station prototype, based on a battery converter as the core component using converters widely available on the market. The battery converter will control the power flow through the charging grid and it has an auxiliary input used to connect it with the power outlet. By using this configuration, the energy management system (EMS) will set the current value extracted from the outlet. The solar inverter will be a current controlled source, connected in parallel with the charging grid. The battery current flow direction and value will also be decided by the EMS. The EV charging power will be, in the best-case scenario, the sum of all the maximum powers, providing in this case a much greater power than the outlet, making fast charging possible in places where otherwise it will not be. The block diagram of the proposed charging station is represented in Fig. 1. The proposed system is primarily based on a single-phase household user power grid with a maximum installed power of $3.6 \mathrm{~kW}$.

If the charging stations are made modular, as operating conditions change or certain technologies become more affordable in terms of cost, users of electric vehicles can reconfigure the system. The different ways in which this system can be used depends on the renewable energy availability, the costs of the technologies used, the season, and the way of using the electric vehicle.

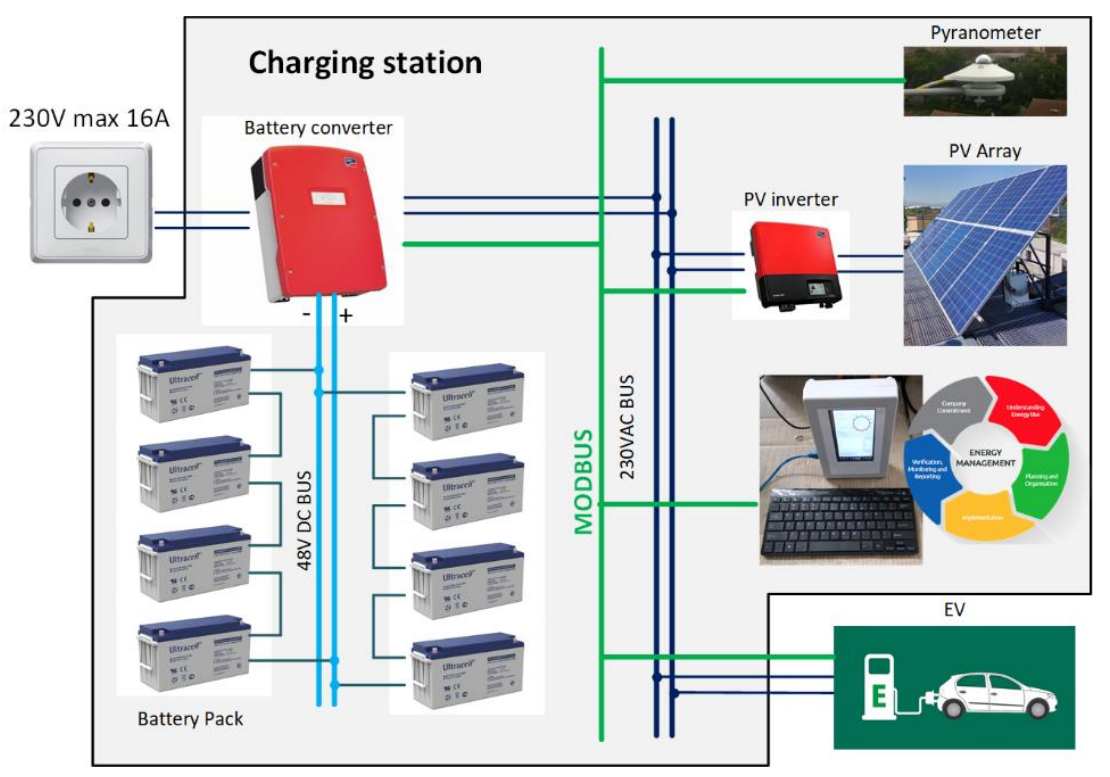

Fig. 1. Proposed charging station with renewable energies 
In the case of the experimental charging station, it was decided to use converters widely available on the market. Thus, the solar inverter used is a Sunny Boy SB3600-TL $(3.6 \mathrm{~kW})$ and the battery converter is Sunny Island type $6.0 \mathrm{H}$ $(4.5 \mathrm{~kW})$, both from SMA. 12 ET-Solar panels of $250 \mathrm{~W}$ were chosen, with the following characteristics: current and voltage at the maximum power point $8.24 \mathrm{~A}$ and $30.34 \mathrm{~V}$ respectively. The BSS is composed of 2 strings of batteries connected in parallel. Each string has 4 VRLA 200AH batteries connected in series for a $48 \mathrm{~V}$ DC bus. The communication network between the centralized management system and the microgrid components is achieved on RS485 using the ModBus protocol.

\section{EV STATION CHARGING MODES}

The charging station voltage and frequency will be imposed by the auxiliary input, in this case the outlet power. Considering the different power flow possibilities and the availability of the power sources, the EV station can operate in either of the following modes. The solar inverter acts as a slave and works as a current source. In Fig.2, $P_{\text {grid }}$ is the power extracted from the power outlet and can be maximum $3.6 \mathrm{~kW}$, $P_{\text {solar }}$ is the power coming from the solar panels and $P_{B S S}$ is the bidirectional power flow charging/discharging the local battery pack. Depending on the operation mode, the value of $P_{B S S}$ can be equal with $P_{\text {grid }}, P_{\text {solar }}$ or a combination of these two values. $P_{E V}$ is the sum of the battery converter $P_{i n v}$ power and solar inverter $P_{\text {solar }}$ power, charging the EV.

\section{A. Mode 1-PV only.}

If there is enough photovoltaic energy available, the electrical vehicle is charged only from the PV system. The charging is done through the DC-AC converter, with maximum power point tracking (MPPT). The battery system and the AC grid are used only to set the AC voltage and frequency. In this mode, the charging power is limited to the available power from the photovoltaic panels and depends on the irradiance, time of day and temperature. This mode of operation is suited for public parking when minimum charging times is not mandatory and during peak consumption hours.

$$
\left\{\begin{array}{l}
P_{E V}=P_{\text {solar }} \\
P_{I N V}=0
\end{array}\right.
$$

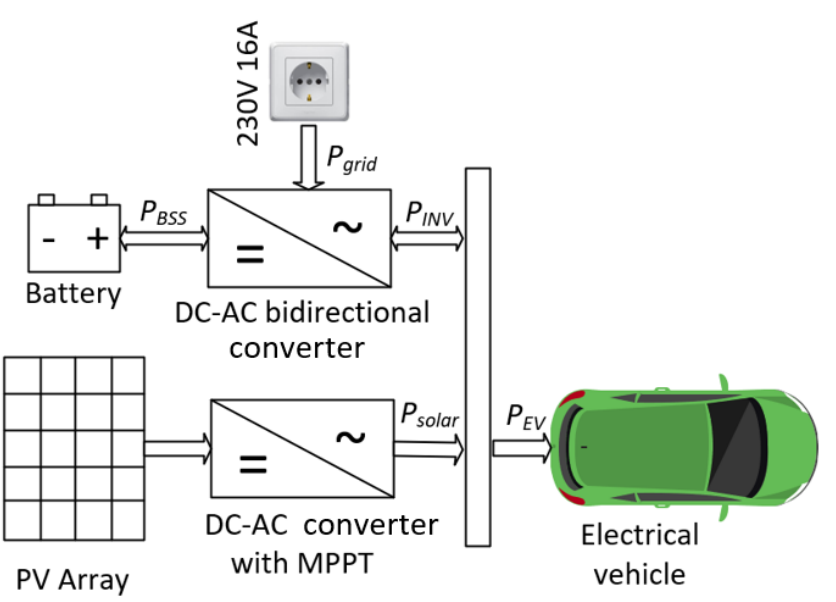

Fig. 2. Charging station block diagram used to describe the operation modes

\section{B. Mode $2-$ Grid only.}

If the local battery is discharged and there is no solar energy available (very low irradiance), the EV can be charged from the grid. In this mode of operation, the charging power is limited to the maximum allowed power from the grid ( $\max$ $3.6 \mathrm{~kW}$, depending on regulations). In this mode, the charging takes longer and it is recommended to be used for charging overnight.

$$
\left\{\begin{array}{l}
S_{B S C} \leq 20 \% \\
P_{\text {solar }}=0 \\
P_{I N V}=P_{\text {grid }}
\end{array} \rightarrow P_{E V}=P_{I N V}\right.
$$

\section{Mode $3-$ Grid and PV.}

In this mode of operation, if there is less available energy from the PV panels (low irradiance), the energy to charge the car can be supplemented from the grid. In this case, the local battery is discharged and cannot be used. This mode is suited when shorter charging times are desired. The maximum power from the grid and the maximum available power from the PV panels can be used to shorten the charging time of the vehicle.

$$
\left\{\begin{array}{l}
S O C_{B S S} \leq 20 \% \\
P_{\text {solar }} \neq 0 \\
P_{I N V}=P_{\text {grid }}
\end{array} \rightarrow P_{E V}=P_{I N V}+P_{\text {solar }}\right.
$$

\section{Mode 4-PV selling energy to the grid.}

In this mode of operation, the local battery is fully charged, and no car is connected to the charging station. The electrical energy produced by the solar panels can be sold to the grid if $P_{\text {grid }}$ is assumed bidirectional.

$$
\left\{\begin{array}{l}
S O C_{B S S} \geq 80 \% \\
P_{E V}=0 \\
P_{\text {grid }}=-P_{I N V}
\end{array} \rightarrow P_{I N V}=P_{\text {solar }}\right.
$$

\section{E. Mode 5-charging the EV from the local battery only}

If the local battery is charged and there is no power available from the PV array, then the EV can be charged only from the local battery. In this case, the grid is used only to set the AC voltage and frequency. In this mode, if the battery converters allow it, the grid can be completely disconnected. The time needed to charge the EV depends on the maximum power of the battery converter and the local battery pack size. In this case, the operation is optimized for the lower charging prices, if it is assumed that the battery was charged beforehand from the solar panels or from the grid at a lower tariff.

$$
\left\{\begin{array}{l}
S O C_{B S S} \in[20 \%, 100 \%] \\
P_{\text {solar }}=0 \\
P_{\text {grid }}=0 \\
P_{I N V}=P_{B S S}
\end{array} \rightarrow P_{E V}=P_{I N V}\right.
$$

\section{F. Mode 6 Local battery and solar system}

In this mode of operation (a fast-charging mode), the energy supplied by the local batteries is supplemented by the 
solar energy. Charging times can be reduced even more or local battery energy can be preserved for future use.

$$
\left\{\begin{array}{l}
S_{B S S} \geq 20 \% \\
P_{\text {solar }} \neq 0 \\
P_{\text {grid }}=0 \\
P_{I N V}=P_{B S S}
\end{array} \rightarrow P_{E V}=P_{I N V}+P_{\text {solar }}\right.
$$

\section{G. Mode 7 Local battery and the grid}

In this mode of operation, if shorter times for charging are desired and no solar energy is available (low irradiance or during the night) the battery energy can be used in conjunction with the grid to charge the EV battery at high power levels.

$$
\left\{\begin{array}{l}
S O C_{B S S} \geq 20 \% \\
P_{\text {solar }}=0 \\
P_{I N V}=P_{B S S}+P_{\text {grid }}
\end{array} \rightarrow P_{E V}=P_{I N V}\right.
$$

\section{H. Mode 8 Local battery, the solar system, and the grid}

Even shorter charging time periods can be obtained if the entire system is used (local battery, grid, and PV). This mode of operation allows the shortest charging time if $\mathrm{P}_{\text {inv }}$ and $\mathrm{P}_{\text {solar }}$ are maximum. Even if the charging time is optimum in this case, the charging price is not, because $\mathrm{P}_{\text {grid }}$ is used at its highest value. This is the most favorable case considering the irradiance during the charging time constant and near its peak value and the SOC of the BSS high enough so that it will not drop under $20 \%$ before the EV is fully charged.

$$
\left\{\begin{array}{l}
S O C_{B S S} \geq 20 \% \\
P_{\text {solar }} \neq 0 \\
P_{I N V}=P_{B S S}+P_{\text {grid }}
\end{array} \rightarrow P_{E V}=P_{I N V}+P_{\text {solar }}\right.
$$

\section{Mode 9 Charging the local battery}

The last mode of operation presented can be used to charge the local battery. It is desirable to charge the local battery from solar energy, reducing in this way the cost of energy; however, there are situations (for example in the winter, with 6-7 days without sunlight) when the charging of the local battery must be done from the grid. Usually this is done overnight to avoid overloading the grid. If necessary, the local battery pack can be charged both from the local grid and from the solar system during the day, when no cars are connected to the station.

$$
P_{B S S}=P_{\text {grid }}+P_{\text {solar }}
$$

\section{MICROGRID SIMULATION MODEL AND RESULTS}

A simulation model in PSIM was developed to test the operation modes of the charging station, Fig. 3.

The model is composed of 3 converters and an AC source to simulate the connection to the grid through the power outlet. The battery converter is bidirectional and can deliver electrical energy to the car when needed.

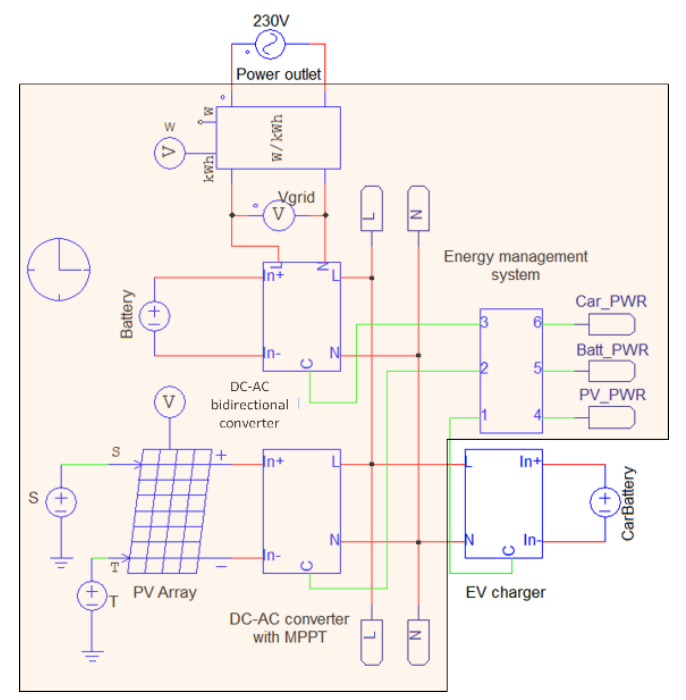

Fig. 3. Charging station simulation model

The battery converter is the master supplying the AC bus of the charging station from batteries or from the $\mathrm{AC}$ outlet depending on the necessary output power.

Because the maximum power extracted from the grid is $3.6 \mathrm{~kW}$ (max 16A), the battery converter will supplement any necessary surplus of power from the batteries. The converter will charge the batteries from the grid, from the solar panels or from both, depending on the availability of the photovoltaic energy.

The solar inverter is a unidirectional current source inverter with MPPT working as a slave. This inverter supplies electrical energy from the PV panels to the AC bus. The car charger is simulated with a unidirectional converter. This converter is also a current source converter. Based on the power levels measured from each converter, an energy management system controls each converter to work in one of the modes detailed above.

A simulation model was developed for each converter but only one is represented in Fig. 4, the others being implemented in a similar way. It is a current source converter, composed of a single-phase bridge topology. It contains a PLL block used for synchronization with the grid. The PLL is a quadrature signal generation PLL (QSG-PLL) implemented based on T/4 transport delay technique [22].

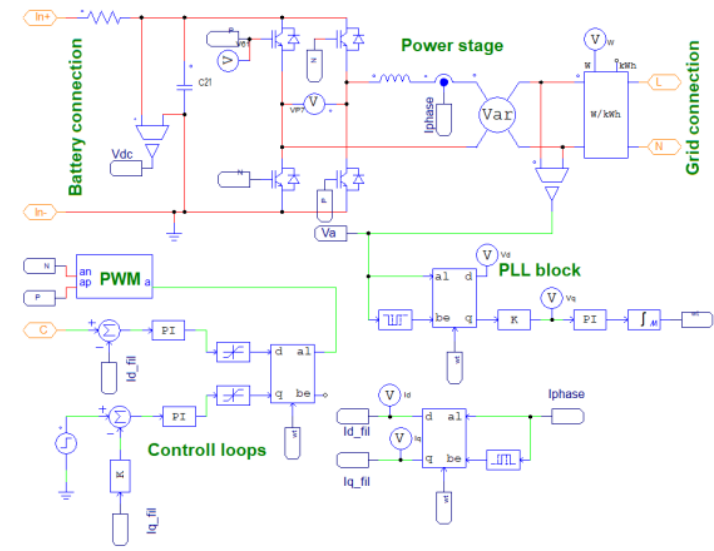

Fig. 4. Current source converter 
This technique allows a vector control approach to be adopted when dealing with single phase systems. The control loops are implemented in rotating DQ frame using the T/4 delay as well. Only the active component of the grid current is controlled.

The waveforms describing the operation of the converter are represented in Fig 5. The first waveform, Fig 5a, is the $I_{d}$ component of the current. The state of the converter changes from supplying an output current of 20A from batteries to the charging station to charge the batteries with 40A. In charging mode, because this current is higher than the one the grid can provide, the difference is supplied from the solar system.

Fig. 5b describes the operation of the PLL block. The grid voltage scaled together with the PLL output are represented. It can be observed that the two waveforms are synchronized. The synchronization is not affected by the change in the output power.

The output current of the converter is represented in Fig $5 \mathrm{c}$. The output current changes its value from $20 \mathrm{~A}$ to $40 \mathrm{~A}$. It can be observed that, because the converter changes state from discharging the batteries to charging the batteries, the output current changes its phase.

The output power is represented in Fig. $5 d$. The positive value shows that the local battery is supplying $3.2 \mathrm{~kW}$ of power to the charging station. The negative value shows that the batteries are charged with $6.2 \mathrm{~kW}$ of power. The waveforms show the proper operation of the bidirectional converter model.

In the following section, all the nine operation modes are described, and they are represented in Fig. 6. In this figure, negative power for the car converter means that the car battery is charging. Concerning the grid, positive power means that energy is taken from the grid and negative power means that power is supplied to the grid from the charging station. For a short simulation time all the operation modes are simulated on a 2 s time interval.

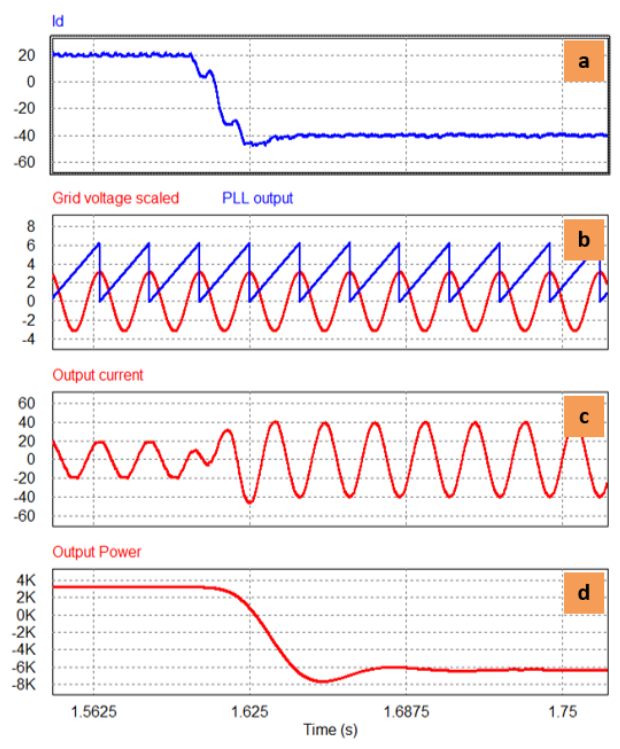

Fig. 5. Operation of the current controlled converter
In mode 1 , the EV is charged only from the PV array with $3.2 \mathrm{~kW}$. This mode is possible only when there is enough solar energy available.

In mode 2, the solar energy is lost, and the car battery is charged from the grid. This mode is used when the local batteries are completely discharged or during nighttime, when the cost of energy is low and there are no constraints for the charging time. The maximum power drawn from the grid is $3.6 \mathrm{~kW}$.

In mode 3, the EV battery is charged from the grid and also from the solar panels; it is assumed that the maxim solar energy in this case is $1.5 \mathrm{~kW}$. In this mode, it is considered that the local battery is discharged or it is not available.

If the car is disconnected and there is enough solar energy, then this energy does not have to be lost; it can be delivered to the grid. In mode 4 , it can be observed that $1.5 \mathrm{~kW}$ of solar power is delivered to the grid.

In mode 5, the car battery is charged only from the local battery with $3.2 \mathrm{~kW}$. The grid and the solar system are considered not to be available.

In mode $6,3.2 \mathrm{~kW}$ of solar power is available and the EV is charged from the local battery and from the solar energy. In this mode, fast charging is possible if enough solar energy is available.

If there is not enough solar energy available, then fast charging mode is available using energy from the grid. In mode 7 , the EV is charged with $6.2 \mathrm{~kW}$.

Mode 8 is the fastest of all and uses all the available sources at maximum power level. In this mode, the EV battery is charged with $9.5 \mathrm{~kW}$.

The last mode of operation deals with charging the local battery. This can be done overnight with energy from the grid or during daytime when there are no EVs connected. The local battery in the current study is charged with $6.2 \mathrm{~kW}$. The highlighted region depicts the fast-charging modes.

\section{CONCLUSIONS}

The current paper presents an EV charging station with renewable energies which includes a battery converter easily found on the market that can be used to reduce the charging time of the EV. The battery converter, together with the grid and the solar system, can be seen as a "charging station". This charging station can be used with EVs that have high power on-board chargers, reducing the charging time. Also, this charging grid can assist high power off-board chargers in areas where the grid power is limited to $3.6 \mathrm{~kW}$. A simulation model was developed to validate the operation of the proposed charging station. The simulation model uses current control bidirectional converters with vector control. Possible operation modes were validated through simulation, demonstrating the effectiveness of such a charging station.

Future work involves testing and validating all the operation modes on a hardware laboratory scale microgrid testbed equipped with a charging station prototype. Different energy management algorithms will be tested. 


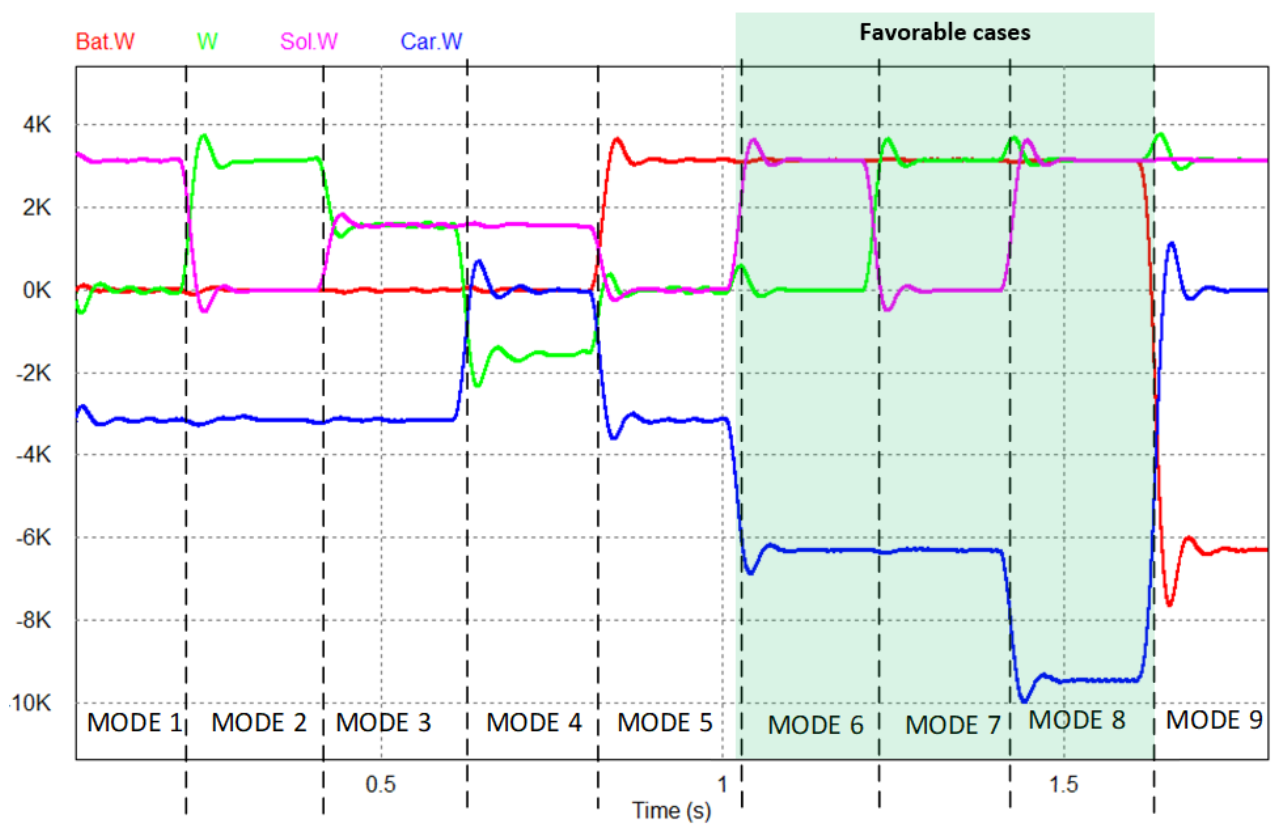

Fig. 6. Simulation of the operation modes

\section{REFERENCES}

[1] A. Matallana et al., "Power module electronics in HEV/EV applications: New trends in wide-bandgap semiconductor technologies and design aspects," Renewable and Sustainable Energy Reviews, vol. 113. Elsevier Ltd, p. 109264, 01-Oct-2019.

[2] S. Sharma, A. K. Panwar, and M. M. Tripathi, "Storage technologies for electric vehicles," Journal of Traffic and Transportation Engineering (English Edition), vol. 7, no. 3. Periodical Offices of Chang- an University, pp. 340-361, 01-Jun-2020.

[3] M. J. Rutherford and V. Yousefzadeh, "The impact of electric vehicle battery charging on distribution transformers," in Conference Proceedings - IEEE Applied Power Electronics Conference and Exposition - APEC, 2011, pp. 396-400.

[4] R. Das, K. Thirugnanam, P. Kumar, R. Lavudiya, and M. Singh, "Mathematical modeling for economic evaluation of electric vehicle to smart grid interaction," IEEE Trans. Smart Grid, vol. 5, no. 2, pp. 712721, Mar. 2014.

[5] R. C. Green, L. Wang, and M. Alam, "The impact of plug-in hybrid electric vehicles on distribution networks: A review and outlook," Renewable and Sustainable Energy Reviews, vol. 15, no. 1. Pergamon, pp. 544-553, 01-Jan-2011.

[6] "Future of EV Charging | IEEE Conference Publication | IEEE Xplore." [Online]. Available: https: //ieeexplore. ieee. org /document/ 9007203. [Accessed: 27-Jun-2021].

[7] R. H. Ashique, Z. Salam, M. J. Bin Abdul Aziz, and A. R. Bhatti, "Integrated photovoltaic-grid dc fast charging system for electric vehicle: A review of the architecture and control," Renewable and Sustainable Energy Reviews, vol. 69. Elsevier Ltd, pp. 1243-1257, 01Mar-2017.

[8] N. Sujitha and S. Krithiga, "RES based EV battery charging system: A review," Renewable and Sustainable Energy Reviews, vol. 75. Elsevier Ltd, pp. 978-988, 01-Aug-2017.

[9] M. T. Hussain, D. N. Bin Sulaiman, M. S. Hussain, and M. Jabir, "Optimal Management strategies to solve issues of grid having Electric Vehicles (EV): A review," Journal of Energy Storage, vol. 33. Elsevier Ltd, p. 102114, 01-Jan-2021.

[10] J. Ping, Z. Yan, and S. Chen, "A Two-stage Autonomous EV Charging Coordination Method Enabled by Blockchain,” J. Mod. Power Syst. Clean Energy, vol. 9, no. 1, pp. 104-113, Jan. 2021.

[11] J. M. Clairand, J. Rodriguez-Garcia, and C. Alvarez-Bel, “Assessment of Technical and Economic Impacts of EV User Behavior on EV
Aggregator Smart Charging," J. Mod. Power Syst. Clean Energy, vol. 8, no. 2, pp. 356-366, Mar. 2020.

[12] K. Zhao, P. Ciufo, and S. Perera, "Rectifier capacitor filter stress analysis when subject to regular voltage fluctuations," IEEE Trans. Power Electron., vol. 28, no. 7, pp. 3627-3635, 2013.

[13] H. L. Li, X. M. Bai, and W. Tan, "Impacts of plug-in hybrid electric vehicles charging on distribution grid and smart charging," in 2012 IEEE International Conference on Power System Technology, POWERCON 2012, 2012.

[14] J. G. Ingersoll and C. A. Perkins, " $2.1 \mathrm{~kW}$ photovoltaic electric vehicle charging station in the city of Santa Monica, California," in Conference Record of the IEEE Photovoltaic Specialists Conference, 1996, pp. 1509-1512.

[15] P. Denholm, M. Kuss, and R. M. Margolis, "Co-benefits of large scale plug-in hybrid electric vehicle and solar PV deployment," J. Power Sources, vol. 236, pp. 350-356, Aug. 2013.

[16] M. E. Kabir, C. Assi, M. H. K. Tushar, and J. Yan, "Optimal Scheduling of EV Charging at a Solar Power-Based Charging Station," IEEE Syst. J., vol. 14, no. 3, pp. 4221-4231, Sep. 2020.

[17] X. Duan, Z. Hu, and Y. Song, "Bidding Strategies in Energy and Reserve Markets for an Aggregator of Multiple EV Fast Charging Stations with Battery Storage," IEEE Trans. Intell. Transp. Syst., vol. 22, no. 1, pp. 471-482, Jan. 2021.

[18] A. Hussain, V. H. Bui, and H. M. Kim, "Optimal Sizing of Battery Energy Storage System in a Fast EV Charging Station Considering Power Outages," IEEE Trans. Transp. Electrif., vol. 6, no. 2, pp. 453463, Jun. 2020.

[19] A. Verma and B. Singh, "Multimode Operation of Solar PV Array, Grid, Battery and Diesel Generator Set Based EV Charging Station," in IEEE Transactions on Industry Applications, 2020, vol. 56, no. 5, pp. 5330-5339.

[20] M. Singh, K. Thirugnanam, P. Kumar, and I. Kar, "Real-time coordination of electric vehicles to support the grid at the distribution substation level," IEEE Syst. J., vol. 9, no. 3, pp. 1000-1010, Sep. 2015.

[21] Ş. Cîrstea, C. Martiş, A. Cîrstea, A. Constantinescu-Dobra, and M. Fülöp, "Current Situation and Future Perspectives of the Romanian Renewable Energy,” Energies, vol. 11, no. 12, p. 3289, Nov. 2018.

[22] R. Teodorescu, M. Liserre, and P. Rodriguez, Grid Converters for Photovoltaic and Wind Power Systems Chapter 10 Control of Grid Converters under Grid Faults. 2011. 